\title{
Problemas de salud y la vulnerabilidad social en pacientes inmigrantes ingresados con patología infecciosa: estudio caso-control
}

\author{
T. Fauraa, F. Garcíab, P. Islaa, C. Lópeza, M. Robauc, A. Moreno, , A. Tricasc, C. Ligeroc, D. Robles, \\ S. Carmonad, J. M. Mirób y J. M. Gatell ${ }^{b}$

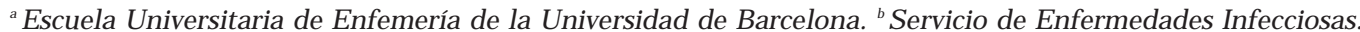 \\ Hospital Clínic. IDIBAPS. Barcelona. 'Dirección de Enfermería. Corporacio Sanitaria Clínic. Barcelona. \\ ${ }^{ } \mathrm{H}$ Hospital Universitari de Bellvitge. L'Hospitalet de Llobregat. Barcelona.
}

\begin{abstract}
Introducción. La necesidad de recursos sanitarios de la población inmigrante ha aumentado en los últimos años. El estudio de los problemas de salud y la vulnerabilidad social planteados durante el ingreso hospitalario de estos pacientes ayudaría a mejorar su cuidado. Pacientes y métodos. Estudio caso-control realizado en el Hospital Clínic de Barcelona. Se incluyeron pacientes inmigrantes ingresados con patología infecciosa de octubre de 2002 a septiembre de 2003 . Los casos fueron apareados por edad, sexo e infección por virus de la inmunodeficiencia humana (VIH). Se evaluaron variables clínicas (visitas a Urgencias, días y número de ingresos, cantidad de procedimientos y fármacos, diagnóstico etiológico y control post alta) y de vulnerabilidad social (utilización de trabajo social, tarjeta sanitaria, cuidador de referencia, consumo de tóxicos, barrera idiomática y alta de enfermería). Resultados. Se estudiaron 102 pacientes ( 51 casos y 51 controles, todos varones). El $56 \%$ estaban infectados por VIH en ambos grupos. EI número de procedimientos diagnósticos o terapéuticos fue mayor en el grupo de inmigrantes $(p=0,02)$, se llegó en menor proporción a un diagnóstico etiológico (el $82 \%$ frente al $98 \%, p=$ 0,021 ) y el número de visitas post alta fue inferior (el $55 \%$ frente al $77 \%, p=0,04)$. Los pacientes inmigrantes tuvieron unos índices de vulnerabilidad social mayores que la población autóctona y en un $35 \%$ de ellos existía una barrera idiomática. Un menor número tenían tarjeta sanitaria (el $63 \%$ frente al $94 \%, p<0,0001$ ) y un número mayor tuvieron necesidad de traslado a un centro sociosanitario (el $16 \%$ frente al $2 \%, p=0,01$ ). Discusión. La vulnerabilidad social de los pacientes inmigrantes influye en una menor obtención del diagnóstico etiológico, mayor número de procedimientos durante la hospitalización y un menor seguimiento posterior al alta.
\end{abstract}

PALABRAS CLAVE: inmigración, vulnerabilidad social, VIH, infección.

Faura T, García F, Isla P, López C, Robau M, Moreno A, Tricas A, Ligero C, Robles D, Carmona S, Miró J M, Gatell J M. Problemas de salud y la vulnerabilidad social en pacientes inmigrantes ingresados con patología infecciosa: estudio caso-control. Rev Clin Esp. 2007;207(5):00-00.
Health problems and social vulnerability in immigrants admitted for an infectious disease: a case-control study

Background. Health resources needed by immigrants have increased steadily in the last few years. Studying health problems and social vulnerability in immigrants would help to improve the health care quality.

Patients and methods. A case-control study performed in the Hospital Clínic of Barcelona. Immigrant patients admitted with infectious diseases from October 2002 to September 2003 were included. Controls were paired by age, gender and HIV infection. Clinical (emergency room attendance, days and number of admission to hospital, amount of clinical procedures and drugs used during the admission, etiological and microbiological diagnosis and post-admission control) and social vulnerability variables (social worker consultation, health care card, relatives or friends caregiver, drug use, language barrier and discharge document of the nurse) were analyzed. Results. One hundred and two patients (51 cases and 51 controls, all of them males) were studied. A total of $56 \%$ were HIV-1 infected in both groups. The number of diagnostic or therapeutic procedures was higher in the immigrant group $(p=\mathbf{0 . 0 2})$, a lower proportion of patients had a final etiologic diagnosis $(82 \%$ vs $98 \%$, p $=0.021$ ) and the number of post-discharge controls was lower $(55 \%$ vs $\mathbf{7 7 \%}, \mathrm{p}=\mathbf{0 . 0 4})$. Immigrants had a higher social vulnerability index than the Spanish population and $35 \%$ could not speak Spanish, French or English. The number of immigrants with health care card was lower $(63 \%$ vs $94 \%, p<0,0001)$ and a higher number needed to be admitted to a social-health care center after discharge $(16 \%$ vs $2 \%, p=0.01)$.

Conclusions. Social vulnerability influences the etiological diagnosis, the number of diagnostic and therapeutic procedures during the admission to the hospital and post-discharge control of immigrant population.

KEY WORDS: immigration, social vulnerability, HIV, infections.
Correspondencia: F. García.

Servicio de Enfermedades Infecciosas.

H ospital Clínic. IDIBAPS.

C/ Villarroel 170.

08036 Barcelona.

Corrreo electrónico: fgarcia@clinic.ub.es

Aceptado para su publicación el 19 de diciembre de 2006.

Las Sras. T. Faura, P. Isla, C. López, M. Robau y A. Tricas han recibido una beca de investigación de la Escuela Universitaria de Enfermería de la Universidad de Barcelona. El Dr. F. García ha recibido una beca de investigación del Institut d'Investigacions B iomèdiques August Pi I Sunyer (IDIBAPS), Barcelona.

\section{Introducción}

El Plan Nacional de Salud 2003 cifra la población inmigrante (legal e ilegal) tributaria de utilizar los servicios sanitarios en Cataluña en un $18 \%$ de la población total ${ }^{1}$. En Cataluña, a pesar de la importancia económica y social del fenómeno de la inmigración, todavía existen pocos datos en el ámbito hospitalario sobre sus nece- 
FAURA T ET AL. PROBLEMAS DE SALUD Y LA VULNERABILIDAD SOCIAL EN PACIENTES INMIGRANTES INGRESADOS CON PATOLOGÍA INFECCIOSA: ESTUDIO CASO-CONTROL

TABLA 1

Definición de las variables estudiadas en los dos grupos

\begin{tabular}{|c|c|}
\hline Variables & Definición \\
\hline \multicolumn{2}{|l|}{ Clínicas } \\
\hline 1. Visitas a Urgencias & Número de visitas a Urgencias del mismo centro entre octubre de 2002 y septiembre 2003 por paciente \\
\hline 2. Ingresos & Número de ingresos en el período descrito por paciente \\
\hline 3. Estancias & Suma de días de hospitalización en el período descrito por paciente \\
\hline 4. Estancia media & Días totales de ingreso dividido por el número de ingresos por paciente \\
\hline 5. Procedimientos & $\begin{array}{l}\text { Suma de pruebas diagnósticas invasivas y/ o procedimientos quirúrgicos realizados durante las distintas } \\
\text { hospitalizaciones por paciente }\end{array}$ \\
\hline 6. Medicamentos & Número de medicamentos prescritos en el informe de alta por paciente \\
\hline 7. Diagnóstico etiológico & Diagnóstico definitivo en el informe de alta. Se han registrado los tres primeros de cada informe \\
\hline 8. Diagnóstico microbiológico & Confirmación microbiológica de los diagnósticos definitivos \\
\hline 9. Control post alta & Controles después del alta \\
\hline \multicolumn{2}{|l|}{ Vulnerabilidad social } \\
\hline 10. Toxicomanías & Consumo de drogas intravenosas, tabaco, alcohol, pastillas y/ o cocaína \\
\hline 11. Alta de enfermería & Indicación de traslado a un centro sociosanitario después del alta \\
\hline 12. Tarjeta sanitaria & Posesión de tarjeta sanitaria. Indica acceso a todos los servicios sanitarios de la red pública \\
\hline 13. Trabajadora social & Consulta a la trabajadora social para pacientes hospitalizados. Indica problemática social asociada \\
\hline 14. Cuidador de referencia & Existencia de pareja o familiar próximo para cuidar al paciente después del alta \\
\hline 15. Barrera idiomática & No hablan ni entienden el idioma español, inglés o francés; solo su propia lengua \\
\hline
\end{tabular}

sidades de salud y sobre las repercusiones en la actividad concreta de los profesionales ${ }^{2}$. En publicaciones recientes ${ }^{3-5}$ se afirma que las poblaciones vulnerables sufren más problemas de salud debido a la falta de recursos y una mayor exposición a riesgos derivados de la pobreza y el bajo nivel de instrucción. Anderson et al $^{6}$ definen población vulnerable como «aquellos grupos sociales formados por personas no caucásicas, con pocos recursos económicos y/ o marginados por falta de instrucción, conducta sexual, estatus de inmigrante y religión o credo».

En el caso concreto de las enfermedades infecciosas se ha descrito que las personas inmigrantes infectadas por el virus de la inmunodeficiencia humana (VIH) podrían tener una supervivencia menor ${ }^{7,8}$. Factores de riesgo prevalentes en la población vulnerable como el consumo de sustancias tóxicas, abandono u errores en la medicación, caquexia y dietas inadecuadas se asocian frecuentemente a los pacientes con infecciones y sida en nuestro medio ${ }^{9}$. Es poco conocido si la barrera idiomática entre los profesionales y los pacientes inmigrantes repercute en una menor adhesión al tratamiento, menor colaboración en los cuidados y en las precauciones basadas en la transmisión y otras conductas que son decisivas para la buena evolución clínica de todos los pacientes. El abordaje en la atención de estos pacientes podría requerir cambios en los cuidados para evitar que la barrera idiomática por sí misma no influya en la realización de un diagnóstico preciso, del tratamiento prescrito y de una adecuada calidad de asistencia ${ }^{10-12}$.

En una unidad de enfermedades infecciosas con 23 camas de un hospital terciario de Barcelona, se observó que los ingresos de pacientes inmigrantes aumentaban progresivamente (hasta un $11 \%$ de la unidad en el mes de septiembre de 2002) y que mostraban un perfil clínico y social diferenciado. Una gran parte estaban infectados por VIH y presentaban carencias, principalmente relacionadas con la dificultad de comunicación, la escasez de recursos económicos y la falta de apoyo social y familiar. El desconocimiento del idioma, junto con la falta de datos de procedencia y de las carencias familiares y sociales referidas, se podría asociar a una dificultad en el manejo del paciente.

Con el objetivo de conocer las características de calidad y de los cuidados recibidos por los pacientes inmigrantes hospitalizados, se realizó un estudio caso-control con pacientes autóctonos analizando las diferencias entre las dos poblaciones.

\section{Pacientes y métodos}

\section{Pacientes}

Estudio tipo caso-control. Los casos estudiados fueron todos los pacientes inmigrantes ingresados con patología infecciosa en el Servicio de Enfermedades Infecciosas durante 1 año (octubre 2002-septiembre 2003), y los controles fueron pacientes autóctonos ingresados también por patología infecciosa y en el mismo período de tiempo. Fueron emparejados 1:1 por edad, sexo e infección por VIH. Se definieron como inmigrantes todos los pacientes no nacidos en España sin distinción de tiempo de permanencia en nuestro país, exceptuando los de menos de un mes de permanencia que se consideraron viajeros ${ }^{13}$. Durante el período de reclutamiento se ingresaron en el Servicio de Enfermedades Infecciosas 59 pacientes inmigrantes (el 7,6\% del total de pacientes inmigrantes ingresados en todo el hospital durante el mismo período). Se excluyeron 8 mujeres por considerar que no presentaban igual grado de vulnerabilidad que los hombres inmigrantes ${ }^{14} \mathrm{y}$, por tanto, se estudiaron 51 varones que se emparejaron por edad, sexo e infección por el VIH con 51 pacientes de nacionalidad española. Se obtuvieron los datos extraídos de los registros médicos, de enfermería y de la trabajadora social incluidos en la historia clínica informatizada.

Se estudiaron 9 variables clínicas y 6 de vulnerabilidad social. Todas ellas se definen en la tabla 1.

\section{Variables clínicas}

El número de visitas a U rgencias, el número de ingresos, los días totales de estancia y el promedio son criterios que informan de la calidad de la atención recibida. El numero de procedimientos invasivos y de medicamentos, indican la comple- 


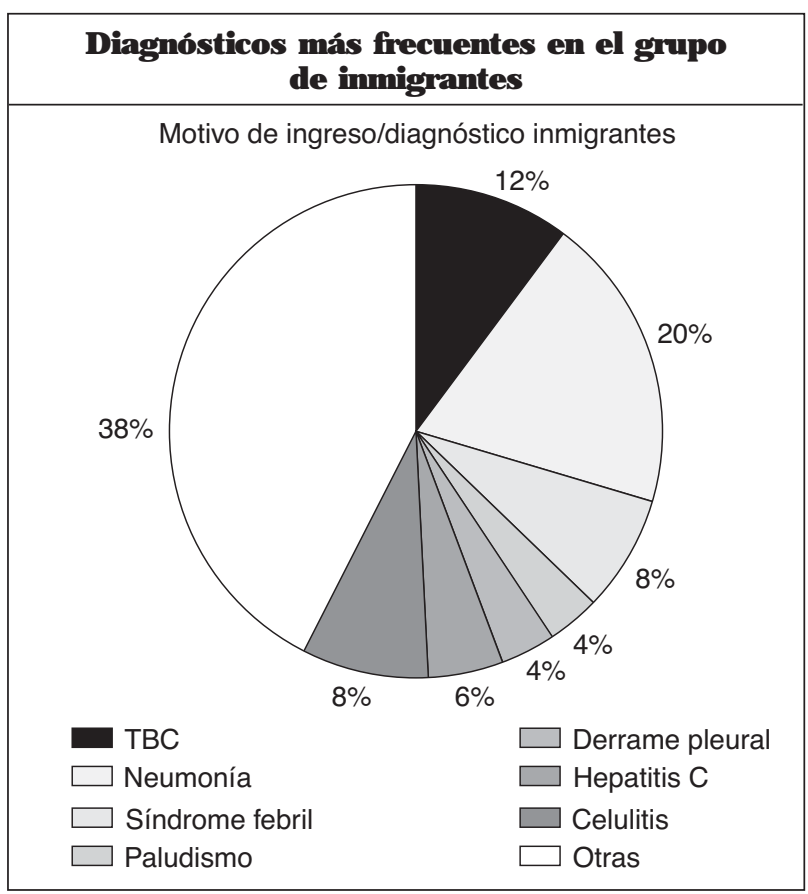

Fig. 1. Diagnósticos más frecuentes en el grupo de inmigrantes.

jidad de la patología tratada, así como el diagnóstico etiológico, el diagnóstico microbiológico y los controles post alta ${ }^{15,16}$.

\section{Variables de vulnerabilidad social}

Se refieren al consumo de tóxicos, el informe de alta de enfermería, que incluye el traslado a otros centros en lugar de al domicilio en caso de que ocurra. Estar en posesión de tarjeta sanitaria en nuestro país indica que pueden acceder a la totalidad de la atención sanitaria existente para todos los ciudadanos y, por tanto, es un factor de menor vulnerabilidad social. La demanda de la trabajadora social, así como la existencia o no de cuidador de referencia y la barrera idiomática son indicadores de menor soporte social y de acceso a la información por desconocimiento del idioma del país ${ }^{17,13}$.

\section{A nálisis estadístico}

Las variables cualitativas se analizaron con una prueba de $\mathrm{Chi}$ cuadrado. Las proporciones fueron comparadas usando las pruebas de McNemar. Las variables cuantitativas con una prueba de $t$ de Student para muestras pareadas en caso de que la variable tuviera una distribución normal y no hubiera diferencias entre las varianzas. Las variables cuantitativas se analizaron con una prueba de Wilcoxon en el caso de que no siguieran una distribución normal o que las varianzas fueran diferentes. Los valores de probabilidad inferiores a 0,05 se consideraron con significación estadística.

\section{Resultados}

Según fuentes del Servicio de Admisiones, en el período estudiado (octubre 2002-septiembre 2003) ingresaron 776 pacientes de lugar de nacimiento distinto a España en el Hospital Clínic de Barcelona. De ellos 59

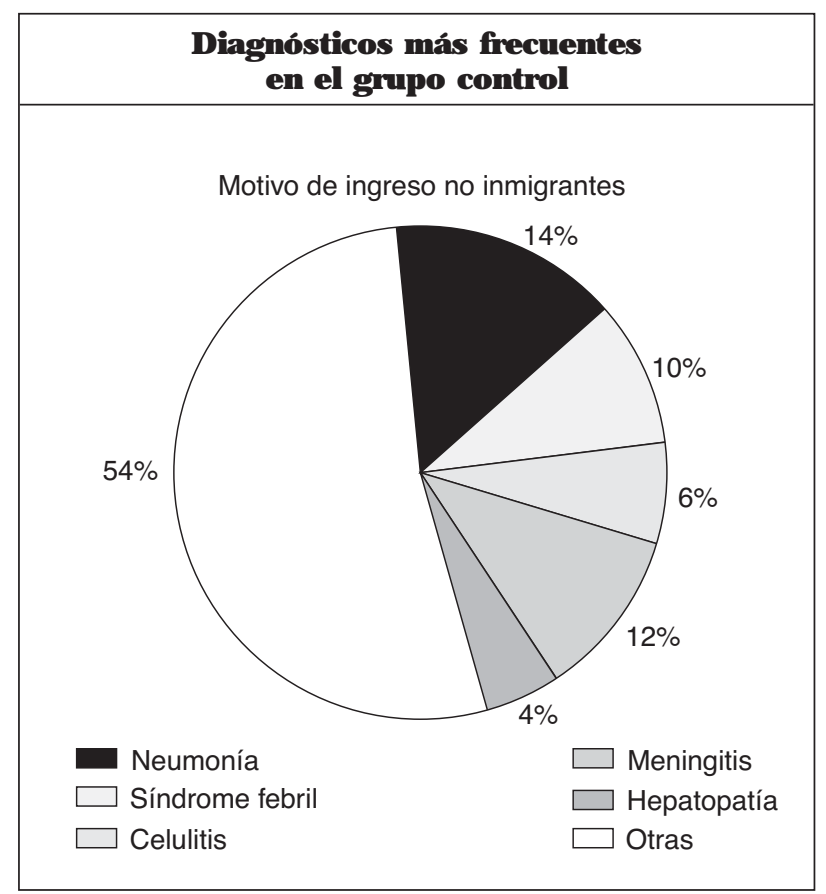

Fig. 2. Diagnósticos más frecuentes en el grupo control.

(el 7,6\% de los ingresos totales) ingresaron en el Servicio de Enfermedades Infecciosas por fiebre o patología infecciosa conocida. Se excluyeron 8 mujeres para tener un grupo más homogéneo socialmente. La cohorte resultante fue de 51 hombres con nacionalidad distinta a la española. Treinta pacientes estuvieron infectados por el VIH en cada grupo. La mediana de edad del grupo de pacientes inmigrantes fue de 37 años (rango intercuartílico: 31-43) y la de los controles de 36 (rango intercuartílico 32-44). El 56\% (30 pacientes en cada grupo) estaban infectados por el VIH, de los cuales el 38\% (19 pacientes) tenían menos de 35 años. Un $23 \%(n=7)$ de los pacientes inmigrantes fueron diagnosticados de infección VIH durante su ingreso hospitalario y 4 de estos 7 pacientes (57\%) continuaron posteriormente en seguimiento. Las patologías mas prevalentes fueron neumonía el $20 \%(n=10)$, tuberculosis (TBC) el $12 \%(n=6)$, celulitis el $8 \%(n=4)$, síndrome febril sin foco el $8 \%(n=4)$, descompensación de cirrosis hepática por hepatitis C el $6 \%(n=3)$, paludismo el $4 \%(n=2)$, derrame pleural el $4 \%(n=2)$ (fig. 1). Los diagnósticos principales en el grupo control fueron neumonía el14\% ( $n=7)$, meningitis el $12 \%$ $(n=6)$, síndrome febril sin foco el $10 \%(n=5)$, celulitis el $6 \%(n=3)$, descompensación cirrosis hepática por hepatitis C el $4 \%(n=2)$ (fig. 2).

Los países de procedencia fueron 22, el más representado fue Pakistán con un $12 \%(n=6)$ seguido de Marruecos con un $8 \%(n=4)$ (fig. 3). Dieciocho pacientes $(35 \%)$ tenían barrera idiomática.

Cuando se realizó el análisis de las variables clínicas no se detectaron diferencias significativas entre el grupo de inmigrantes y autóctonos en el número de visitas a Urgencias, número de ingresos, días de estancia totales, estancia media por ingreso y número de medicamen- 
FAURA T ET AL. PROBLEMAS DE SALUD Y LA VULNERABILIDAD SOCIAL EN PACIENTES INMIGRANTES INGRESADOS CON PATOLOGÍA INFECCIOSA: ESTUDIO CASO-CONTROL

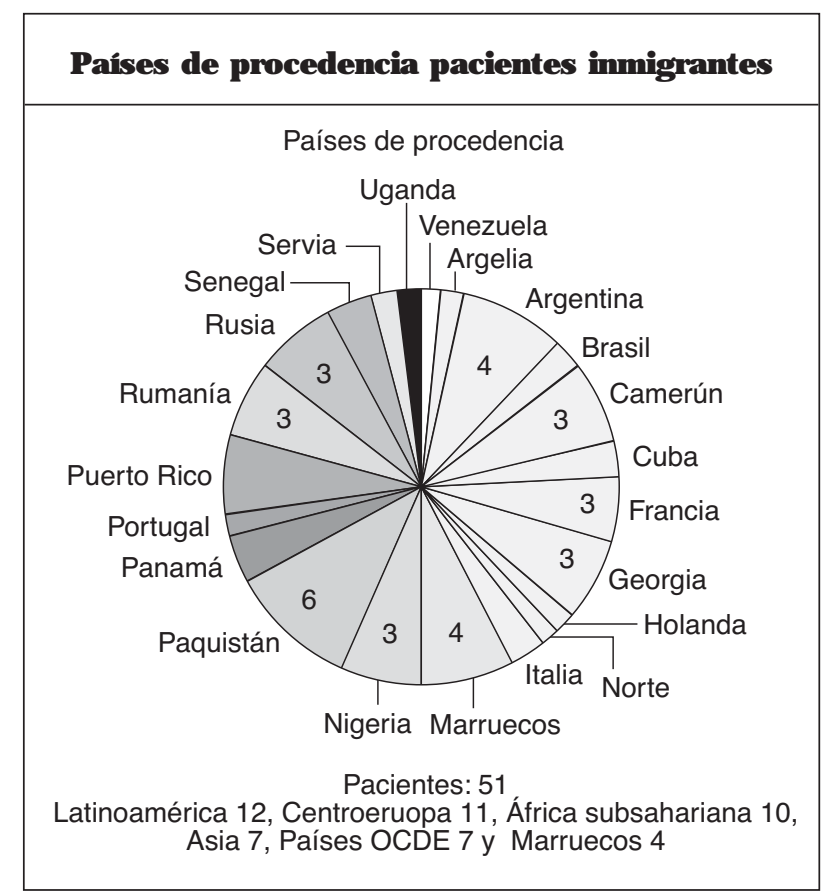

Fig. 3. Países de procedencia de los pacientes inmigrantes.

tos prescritos al alta. Por el contrario, el número de procedimientos diagnósticos invasivos fue significativamente mayor en el grupo de inmigrantes (mediana de 1 frente a 0 en los controles) ( $p=0,02)$. A pesar de ello, en un número menor de inmigrantes se llegó a un diagnóstico etiológico (el $82 \%$ en el grupo de inmigrantes frente al $98 \%$ en el grupo de pacientes autóctonos, $p=0,021$ ). Por último, el número de visitas post alta fueron inferiores en los pacientes inmigrantes (el 55\% en el grupo de inmigrantes frente al $77 \%$ en el grupo de pacientes autóctonos, $p=0,04$ ) (tabla 2).

Los pacientes inmigrantes tuvieron unos índices de vulnerabilidad social mayores que la población autóctona, a pesar que sólo en un 35\% de ellos existía una barrera idiomática. Un menor número de pacientes inmigrantes tenía tarjeta sanitaria (el $63 \%$ en el grupo de casos frente al $94 \%$ en el grupo de controles, $\mathrm{p}<0,0001$ ) o cuidador de referencia (el $27 \%$ en el grupo de casos frente al $96 \%$ en el grupo de controles, $p<0,0001$ ) respecto a la población autóctona, mientras que la necesidad de intervención de la trabajadora social (el $18 \%$ en el grupo de casos frente al $2 \%$ en el grupo de controles, $p=0,04)$ y la necesidad de traslado a un centro sociosanitario después del alta (variable alta de enfermería) (el $16 \%$ en el grupo de casos frente al $2 \%$ en el grupo de controles, $p=0,01$ ) fue superior en el grupo de inmigrantes. Por último, no existieron diferencias significativas entre ambos grupos en el consumo de tóxicos (tabla 3).

\section{Discusión}

En los últimos años la proporción de pacientes con patología infecciosa, y en especial los infectados por
TABLA 2

Resultados de las variables clínicas para cada grupo

\begin{tabular}{|c|c|c|c|}
\hline Variables & $\begin{array}{c}\text { Grupo control } \\
(n=51) \\
\text { Mediana } \\
\text { (rango } \\
\text { intercuartílico) }\end{array}$ & $\begin{array}{c}\text { Grupo inmigrante } \\
\text { (n = 51) } \\
\text { Mediana } \\
\text { (rango } \\
\text { intercuartílico) }\end{array}$ & $\mathbf{p}$ \\
\hline Edad (años) & $36(32-41)$ & $37(31-43)$ & \\
\hline Visitas a Urgencias (n. $\stackrel{0}{ })$ & \multirow{2}{*}{$\begin{array}{l}1(1-2) \\
1(1-2)\end{array}$} & $1(1-2)$ & 0,87 \\
\hline Ingresos (n. $\stackrel{0}{)})$ & & $1(1-2)$ & 0,29 \\
\hline Estancias (días) & $14(6-30)$ & $10(5-20)$ & 0,80 \\
\hline Estancia media (días) & $9(5-17)$ & $8(4-16)$ & 0,92 \\
\hline Procedimientos ( $\left.\mathrm{n} . \mathrm{o}^{\prime}\right)$ & $0(0-2)$ & \multirow{2}{*}{$\begin{array}{l}1(1-3) \\
3(1,5-7)\end{array}$} & 0,02 \\
\hline \multirow[t]{2}{*}{ Medicamentos (n. $\stackrel{0}{ })$} & $4(1,5-5,5)$ & & 0,69 \\
\hline & No & No & \\
\hline Diagnóstico etiológico & 50 & 42 & 0,021 \\
\hline Diagnóstico microbiológico & 28 & 19 & 0,10 \\
\hline Control post alta & 39 & 28 & 0,04 \\
\hline
\end{tabular}

el VIH no nacidos en España, es cada vez mayor, debido a un aumento progresivo de la población inmigrante. En el año 2003 el número de inmigrantes se cifro en un $18 \%$ de la población total ${ }^{1}$. En España, el 12,3\% de los pacientes con sida notificados en el 2003 eran extranjeros $^{8}$. En el Servicio de Infecciones del Hospital Clínic de Barcelona, los inmigrantes representaron el 7,6 del total de extranjeros ingresados en el centro. En nuestra cohorte de 51 varones inmigrantes con patología infecciosa, los pacientes procedían de países muy variados, con predominio del continente africano seguidos de Latinoamérica y países centroeuropeos. Esta distribución está en línea con informes de inmigración en países europeos en los que los inmigrantes proceden mayoritariamente de África, y se diferencia de los realizados en otros centros de la península ibérica. La gran dispersión de países de procedencia constituye un hallazgo y puede ser la causa de la percepción de sensación de incomunicación o de comunicación no fluida con los pacientes.

Los inmigrantes con infección por el VIH son generalmente muy jóvenes ${ }^{18,19}$, dato comprobado en nuestro estudio. La prevalencia de mujeres fue muy baja, lo que puede ser debido a que socialmente las mujeres inmigrantes son todavía más vulnerables que los hombres y no tienen el mismo acceso a los recursos sanitarios, a pesar de tener igual o mayor nivel de patología. De hecho, se ha publicado que la prevalencia de infección por VIH es superior en las mujeres inmi-

TABLA 3

Resultados de las variables de vulnerabilidad social para cada grupo

\begin{tabular}{|c|c|c|c|c|c|}
\hline \multirow[t]{2}{*}{ Variables } & \multicolumn{2}{|c|}{$\begin{array}{l}\text { Grupo control } \\
\qquad(n=51)\end{array}$} & \multicolumn{2}{|c|}{$\begin{array}{l}\text { Grupo inmigrante } \\
\qquad(\mathrm{n}=51)\end{array}$} & \multirow[t]{2}{*}{$\mathbf{p}$} \\
\hline & Sí & No & Sí & No & \\
\hline Toxicomanías & 26 & 25 & 25 & 28 & 0,66 \\
\hline Alta de enfermería & 1 & 50 & 8 & 43 & 0,01 \\
\hline Tarjeta sanitaria & 48 & 3 & 32 & 19 & $<0,0001$ \\
\hline Trabajadora social & 1 & 50 & 9 & 42 & 0,021 \\
\hline Cuidador de referencia & 49 & 2 & 14 & 37 & $<0,0001$ \\
\hline Barrera idiomática & No & No & 18 & 33 & \\
\hline
\end{tabular}


grantes que en las mujeres autóctonas ${ }^{20}$, por lo que al menos las consultas por esta patología infecciosa en las mujeres deberían ser más elevadas.

Las variables clínicas analizadas demuestran una mayor dificultad para llegar al diagnóstico etiológico en el grupo de inmigrantes, a pesar de que en estos pacientes se realice un mayor número de procedimientos. Este hecho podría estar relacionado con la barrera idiomática, aunque no exclusivamente, ya que sólo una tercera parte de los inmigrantes tuvieron dificultades de comunicarse con el personal sanitario. Este hecho también podría justificarse por la mayor prevalencia de enfermedades, fundamentalmente de tipo infeccioso, que existen en áreas de menor desarrollo económico, aunque otros factores culturales o médicos (patologías no conocidas en nuestro entorno) podrían explicar estas diferencias. A simismo, la mayor probabilidad en los inmigrantes de no alcanzar un diagnóstico etiológico permite inferir que podría existir un número mayor de recaídas o complicaciones, que se detectaran 0 no, en función de la frecuencia de visitas al hospital o centro de Atención Primaria. En estudios previos $^{21}$ se ha comunicado un retraso en la asistencia de los inmigrantes infectados por el VIH (valores de linfocitos CD4 inferiores a los pacientes autóctonos). Por desgracia, en nuestro estudio las características de la información no nos permiten disponer de datos que posibiliten analizar los posibles retrasos en el diagnóstico de VIH o en la evolución clínica. Merece la pena también destacar el dato de que más de la mitad de los pacientes inmigrantes que ingresaron por cualquier motivo en una unidad de infecciosos estuvieran infectados por $\mathrm{VIH}$, aun cuando la patología que motivara su ingreso no fuera ninguna relacionada con esta infección por VIH. Este hecho pone de manifiesto la sobrecarga de recursos médicos que el cuidado de estos pacientes implica para el sistema público sanitario, y la importancia de incluirlos en programas de seguimiento adecuados con fines epidemiológicos y clínicos.

Clásicamente diferentes estudios han puesto de manifiesto la desigualdad en salud en relación con la vulnerabilidad social ${ }^{22}$. En esta línea nuestro trabajo coincide con el modelo diseñado por Flaskerud y Winslow ${ }^{13}$ donde se afirma que las poblaciones vulnerables sufren más problemas de salud debido a la falta de recursos y una mayor exposición a riesgos derivados de la pobreza y el bajo nivel de instrucción.

En nuestro estudio, aunque el número de visitas al hospital y a U rgencias eran similares en los pacientes inmigrantes que en la población autóctona, el número de visitas post alta programadas de pacientes del grupo de casos fueron menores y en muchas ocasiones ni existían registros posteriores al ingreso. Por tanto, se podía pensar que se habían trasladado a otro lugar 0 acudido a otros centros. Según algunos autores, el factor de la inestabilidad laboral y de vivienda esta muy ligado al buen seguimiento post ingreso ${ }^{19,21}$. Se confirmó que los inmigrantes tienen un menor acceso a los recursos sanitarios en nuestro país debido a que la tarjeta sanitaria es la puerta de acceso a todos ellos y una tercera parte no disponían de ella. Por último, la mayor demanda a la trabajadora social por el grupo de inmigrantes indica una mayor vulnerabilidad social $y$, por tanto, una potencial demanda de centros sociosanitarios o residencias para pacientes sin domicilio fijo ni cuidador responsable, como de hecho así se confirma en nuestro trabajo.

La principal limitación de este trabajo es el reducido número de pacientes inmigrantes y la posibilidad que los enfermos estudiados en nuestro entorno no sean del todo representativos de la población inmigrante residente en Barcelona ${ }^{23,24}$. Por otra parte, el hecho de que sea retrospectivo no permite completar adecuadamente algunas informaciones importantes. Estudios prospectivos con pacientes de ambos sexos que analicen un mayor número de variables podrán aportar mayor información que ayuden a implementar medidas de control futuras más relevantes.

En conclusión, la población inmigrante comporta un número destacado de los enfermos atendidos en un Servicio de Infecciones de un Hospital Universitario. Se comprobó dificultades de comunicación en una tercera parte de los pacientes, en un mayor número de ellos no se alcanzó un diagnóstico etiológico definitivo y en menor número acudieron a controlarse tras el alta hospitalaria. Una mayor vulnerabilidad social de la población inmigrante podría ser una de las causas que originen estos problemas. A pesar de ser una población joven es destacable la necesidad de recursos sociosanitarios de larga estancia. Sería necesario introducir medidas dirigidas a los inmigrantes durante su hospitalización para evitar las dificultades de diagnóstico y seguimiento de estos pacientes. Paralelamente, cualquier mejora en la vulnerabilidad social de los inmigrantes influiría decisivamente en una mejor atención sanitaria.

\section{BIBLIOGRAFÍA}

1. Centro Nacional de Epidemiología. Vigilancia Epidemiológica del Sida en España. Situación a 30 de junio 2003. Bol Epidemiol Semanal. 2003;11:293-7. 2. Berra S, Elorza Ricart J M, Bertomeu N, Hausmann S, Serra-Sutton V, Rajmil L. «Necessitats en salud i utilització dels serveis sanitaris en la població immigrant a Catalunya. Revisió exhaustiva de la literatura científica. Mayo 2004. Agencia d'A valuacio de Tecnologia i Recerca Medica (AATRM). Gene2004. A gencia d A valuacio de Tecr
ralitat de Catalunya Barcelona.

3. Editorial: Al. loctons i autòctons Informatiu Agencia d'A valuació de Tecnología i Recerca Mèdiques. 2004;(34):1-2

4. Wolfers I, Verghis S, Marin M. Migration, human rights and health. Lancet. 2003;362:2019-20.

5. Flaskerud J H, Lesser J,Dixon E, Anderson N, Conde F, Kim S, et al. Health Disparities A mong Vulnerable Populations: Evolution of Knowledge Over Five Decades in Nursing Research Publications. Nursing Research 2002; $51(2): 74-85$

6. Anderson L, Fullilove M, Scrimshaw S, Fielding J, Norman J, Zaza S, et al. A framework for evidenced-based reviews of intervention for supportive social environements. Annals of the New York Academy of Sciences. 1999; 896:487-9.

7. Darbes L, Kennedy G, Peersman G, Rutherford G, Zohrabyan L. Behavioural interventions for decreasing HIV infection in racial ethnic minorities in high-income economies (Protocol for a Cochrane Review). The Cochrane Library. Chichester, UK: J ohn Wiley \& Sons, Ltd. 2004:2:1-6. Disponible en: http:// 212.49.218.200/ newgenClibPlus/ ASP/ printD ocument ble en: http:

8. Ramos J M, et al. Características clínicas y epidemiológicas de la infección por el VIH en extranjeros en Elche, España $(1992,2003)$. Enferm Infec Microbiol Clin. 2005;23(8):469-73.

9. Kindelán J M, del Amo J, Martín Ruiz C, Saiz de la Hoya P, Pulido F, Laguna $\mathrm{F}$ (GESIDA). Tratamiento de la infección por VIH en pacientes con problemática psicosocial. Enferm Infecc Microbiol Clin. 2002;20 Supl 2:19-28. 10. Faura $T$, Fernández $E$, Font $C$, Ligero $C$, Robau $M$, Tricas $A$. Registro de procedencia sociocultural de pacientes atendidos en un hospital de agudos. Estuprocedencia sociocultural de pacientes atendidos en un hospital de agudos. Estude Investigación Cualitativa en Salud. Barcelona, 5 y 6 de febrero 2004. 
FAURA T ET AL. PROBLEMAS DE SALUD Y LA VULNERABILIDAD SOCIAL EN PACIENTES INMIGRANTES INGRESADOS CON PATOLOGÍA INFECCIOSA: ESTUDIO CASO-CONTROL

11. National Institute of Nursing Research (NIH). Mission Statement and Strategic Plan on Reducing Health Disparities. 200:10-15. Bethesda MD. Disponible en: http:// www.nih.gov/ ninr/ diversity/ mission.htm/

12. Dickelmann N, Ironside PM. Developing a science of nursing education. Innovations with research. J ournal of Nursing Education. 2002;41:379-80. 13. Flaskerud $\mathrm{J} \mathrm{H}$. Winslow BJ . Conceptualazing vulnerable populations health related research. Nursing Research. 1998;47(2):69-78.

14. Bollini P, Sien H. No real progress towards equity; $H$ ealth of immigrants 14. Bollini P, Sien H. No real progress towards equity; H ealth of immigrants
and ethic minorities on the eve of the year 2000 . Social Science and Mediand ethic minorities on the
cine. 1995;41(6):819-28.

cine. 1995; 41 (6):819-28.
15. Briss PA, Zaza S, Pappaioanou M, Fielding J , Wright-De Aguero L, Truman $\mathrm{BI}$, et al. Developing an evidence-based Guide to Community Preventive Services-Methods. American J ournal of Preventive Medicine. 2000;18(1s):35-43. 16. Arcelay A, Lorenzo S, Bacigalupe M, Mira JJ, Palacio F, Ignacio E, et al. A daptación de un Modelo de Gestión de Calidad Total al sector sanitario. Revista de Calidad Asistencial. 2000;15(3):70-9.

17. Llacer A, Del Olmo J , Castillo S, Belza MJ. Salud e inmigración; a pro17. Llacer A, Del Olmo , Castillo S, Belza
pósito del sida. Gac Sanit. 2001;15:197-9.

18. Valleroy LA, Mac Kellan DA, Karon J M, et al. HIV prevalence and asociates risks in young men who have sex with men. Young Men's Survey Study Group. J ournal of the American Medical Association. 2000;284:198-204.
19. Palella FJ J r, Delanney KM, Moorman AC, et al. Declining Morbidity and mortality among patients with advanced human immunodeficiency virus infection. H IV O utpatient Study Investigators. N engl J Med 1998; 338:853-860

20. Nyamathi A, Flaskerud J H. HIV-risk behaviors and mental health characteristics among homeless or drug-recovering women and their closest sour-

ces of social support. Nursing Research. 1997;46 (3):133-7.
21. Kelly AG, et al. Racial and Gender Disparities in Receip of Highly Active Antirretroviral Therapy Persist in a Multistate Sample of HIV Patients in 2001. J Acquir Immune Defic Syndr. 2005;38(1):96-101.

22. H aour-Knipe M, Fleury F, Duboid-Arber F. HIV/AIDS prevention for migrants and ethnic minorities: three phases of evaluation. Social Science $\&$ Medicine. 1999;(49):1357-72.

23. Moreno-Cuerda VJ, Morales-Conejo M, Rubio R. Causas de admisión hospitalaria en pacientes con infección por VIH en el área 11 de Madrid. Rev Clin Esp. 2006;206:359-60.

24. Brun Romero FM, Martín A spas A, Guerrero Sánchez F, Montes de O ca Arjona M, Soto Cárdenas MJ , Girón González J A. Cambios en las admisiones y mortalidad en el Servicio de Medicina Interna de un $\mathrm{H}$ ospital de tercer nivel (1996-2000). Influencia de las modificaciones en el tratamiento de la infección de VIH. Rev Clin Esp. 2005;205:51-6. 